\title{
Body Composition and Morphological Limbs Asymmetry in Competitors in Six Martial Arts
}

\author{
Composición Corporal y Asimetría Morfológica de \\ Miembros en Competidores en Seis Artes Marciales
}

Lucia Mala$^{1}$; Tomas Maly ${ }^{1}$; Lee Cabell²; Pavol Cech ${ }^{3}$; Mikulas Hank ${ }^{1}$; Klara Coufalova ${ }^{1}$ \& Frantisek Zahalka ${ }^{1}$

MALA, L.; MALY, T.; CABELL, L.; CECH, P; HANK, M.; COUfALOVA, K. \& ZAHALKA, F. Body composition and morphological limbs asymmetry in competitors in six martial arts. Int. J. Morphol., 37(2):568-575, 2019.

SUMMARY: The aim of this study was to compare body composition (BC) and morphological symmetry differences among elite athletes $(\mathrm{n}=132)$ in six martial arts (judo, karate, fencing, wrestling, taekwondo, kickboxing). Multivariate analysis of variances (MANOVA) was used to compare the following variables: absolute (FFM) and relative $\left(\mathrm{FFM}_{\text {rel }}\right)$ value of fat free mass, percentage of fat mass (FMp), bone mass, protein mass, basal metabolic rate, absolute (TBW) and percentual (TBWp) value of total body water, segmental proportion of muscle mass, phase angle, and percent-age differences between the upper and lower limbs. MANOVA revealed significant differences in BC among the groups $\left(\lambda=0.01, \mathrm{~F}_{104,256}=10.01, \mathrm{p}<0.01, \eta \mathrm{p}^{2}=0.67\right)$. No significant differences were observed for $\mathrm{FM}$, $\mathrm{FFM}_{\mathrm{rel}}$, and TBW $(\mathrm{p}>0.05)$. In all other BC variables, significant differences were detected $(\mathrm{p}<0.05)$. The comparison of paired differences between limbs revealed significantly greater fluid volume in the preferred arm compared to the non-preferred arm in karate and fencing athletes. Significant differences $(\mathrm{p}<0.05)$ in favour of the preferred leg in all combat athletes were detected. This study revealed morphological differences among practitioners of different martial arts and between paired limbs.

KEY WORDS: combat sports, morphological asymmetries, elite athletes, fat mass, fat free mass.

\section{INTRODUCTION}

There is a need to determine the factors that enable high-level athletes to maximize their performance (Krzykala, 2012). The main aim in sports is to achieve the best possible competitive result, and this is the main motivation for training (technical, tactical, physical, and psychological) (Dopsaj et $a l ., 2017)$. In order to achieve desired results in sports, it is necessary for the athlete to possess certain characteristics, among which body structure is greatly important. Body compartments, among other factors, play an important role in physical performance (Mala et al., 2015a). The body structure of an athlete is vital in some sports, while in other sports, such as wrestling, boxing, judo, karate, and rowing, it plays a limited role because competitors are divided into weight categories (Dopsaj et al.). Body composition (BC) is a key consideration in the physical makeup of combat sport athletes. One aim of $\mathrm{BC}$ assessment is to differentiate and quantify the different body compartments (Sutton et al., 2009).

Possessing a body morphology that is suitable for a particular sport is an advantage for the athlete. Moreover, the athlete's body can change to match the optimal morphology for the given sport with appropriate training. Training can also be used to assess the symmetry of active mass distribution in individual segments to minimize the risk of injury (Mala et al., 2014). Due to the explosive activities performed in martial arts, excess adipose tissue acts as a "dead weight" when the body must be repeatedly lifted against gravity during locomotion and jumping (Reilly, 1996). As such, excess adipose tissue decreases performance and increases energy demands. In combat sports, morphological and body structure parameters should be assessed because they may significantly affect the techniques chosen by the athlete during a fight (Kasum \& Dopsaj, 2012).

In the literature, optimal BC has been assessed in the context of martial arts. The following areas have been investigated: weight categories, the optimal proportion of fat free mass and fat mass (Franchini et al., 2011; Mala et al., 2015b), the dimensions of body segments (Franchini et al.), dietary habits, and the effects of fast body weight loss on sport

\footnotetext{
${ }^{1}$ Charles University in Prague, Faculty of Physical Education and Sport, Czech Republic.

${ }^{2}$ Arkansas Tech University, Department of Health and Physical Education, United States.

${ }^{3}$ University of Presov, Faculty of Sports, Slovakia.
} 
performance (Ljubisavljevic et al., 2015; Mala et al., 2016). Only a few studies have covered morphological symmetry and asymmetry in the bodies of athletes (Maly et al., 2016; Rauter et al., 2017) and specifically, morphological asymmetry among athletes competing in combat sports (Dopsaj et al.; Krstulovic et al., 2017; Mala et al., 2017).

Long-term preferred and uncompensated loads on one side of the body may lead to asymmetry and the dominance of one leg or hand over the other, which can be the result of preexisting limb preferences (footedness and handedness). When this situation is repeated over many years, during which the athlete practices repetitive asymmetric loading, some morphological and function asymmetry can develop (Maly et al., 2017). Even if training is conducted correctly, the physical stress imposed by the discipline could be strong enough for athletes to inevitably develop a certain degree of functional and morphological asymmetry as the result of adaptive changes on the dominant side (Vad et al., 2004).

Long-term asymmetrical loading has been shown to cause differences in bone mineral composition and density between the dominant and non-dominant arms of racquet sport players (Kannus et al., 1995). Arm asymmetry has also been observed in professional tennis players, with approximately $20 \%$ more bone mineral content and muscle mass observed in the dominant arm (Calbet et al., 1998). These maladaptive effects occur in young athletes as well as elite and adult athletes. Mala et al. (2017) reported a significantly higher proportion of muscle mass in the preferred limb (upper and lower limb) in young judo athletes $(\mathrm{n}=39$, age $=12.08$ years $)$. Although many studies have focused on BC and morphological/physiological asymmetry in athletes, none has investigated morphological asymmetry among high-level athletes who participate in different martial arts.

The aim of this study was to present the differences in morphological body symmetry among different martial arts competitors and to compare the effects of the type of martial art on eventual asymmetry in order to minimize the risk of injury and to examine the suitability of assessing morphological asymmetry without using more detailed methods.

\section{MATERIAL AND METHOD}

Subjects. Elite male $(\mathrm{n}=132)$ competitors in six different combat sports, namely judo $(\mathrm{J}: \mathrm{n}=19)$, karate $(\mathrm{K}: \mathrm{n}=29)$, fencing $(\mathrm{F}: \mathrm{n}=22)$, wrestling $(\mathrm{W}: \mathrm{n}=25)$, taekwondo (T: $\mathrm{n}=$ $20)$ and kickboxing (Ki: $n=17)$, agreed to participate in the present study. The performance level profiles of the groups are shown in Table I and the basic anthropometric parameters are stated in the Results section (Table II). The athletes did not engage in any strenuous physical exercise for 2 days prior to the evaluation. The procedure of the study was explained to all athletes and signed informed consent was collected before the assessments were performed. The research was approved by the ethical committee of the Faculty of Physical Education and Sport, Czech Republic.

\section{Procedures}

Anthropometric measurement. Anthropometric data were recorded before $\mathrm{BC}$ assessment. Body height $(\mathrm{BH})$ was measured using a digital stadiometer (SECA 242, Hamburg, Germany) and body mass (BM) using a digital scale (SECA 769, Hamburg, Germany).

Assessment of body composition. Body composition data were recorded under the same conditions in the morning: the participants used no medications and did not reduce their body weight prior to the measure-ment,neither radically nor in the long-term before competitions. To assess whole-body bioimpedance, we used a multi-frequency Tanita MC-980MA bioimpedance analyser (Tanita Corp., Tokyo, Japan).

The following variables were recorded: body height $(\mathrm{BH})$, body mass $(\mathrm{BM})$, body mass index (BMI), fat free mass $(F F M)$, relative value of fat free mass $\left(F^{2} M_{\text {rel }}\right)$ percentage of fat mass $\left(\mathrm{FM}_{\mathrm{p}}\right)$, total body water (TBW), percentage of total body water $\left(\mathrm{TBW}_{\mathrm{p}}\right)$, extracellular water $(\mathrm{ECW})$, intracellular water (ICW), bone mass (BM), segmental fluid distribution for trunk, upper and lower limb as well as their differences between paired limbs (preferred vs. non-preferred).

Statistical analysis. For all dependant variables, we calculated basic descriptive statistics: central tendency (mean), variability (standard deviation), and shape (skewness, kurtosis). The assumptions for using a parametric test were satisfied, and differences in the observed dependant variables among the groups were assessed using multivariate analysis of variance (MANOVA). We used multiple comparisons of means (Bonferroni's post-hoc test) to compare differences in particular parameters among the groups. The comparison of segmental fluid distribution between limbs was performed using Student's t-test for dependent variables, which was preceded by analysis of variance (ANOVA) based on an F-test.

For all analyses, the statistical significance level was set at $p=0.05$ and the effect size was assessed using the "partial Eta square" coefficient $\left(\eta p^{2}\right)$. Statistical analysis was performed using IBM ${ }^{\circledR}$ SPSS $^{\circledR}$ v24 (Statistical Package for Social Science, Inc., Chicago, IL, 2012). 


\section{RESULTS}

Multilevel analysis of variance revealed significant differences in body composition parameters among the groups $\left(\lambda=0.01, \mathrm{~F}_{104.256}=10.01, \mathrm{p}<0.01, \eta \mathrm{p}^{2}=0.67\right)$.

Anthropometric parameters. Comparison of basic parameters revealed significant differences in age among the groups $\left(\mathrm{F}_{5,125}=4.06, \mathrm{p}=0.00, \eta \mathrm{p}^{2}=0.14\right)$ (Table II). Bonferroni's post-hoc test indicated a significant difference between fencers and wrestlers/taekwondo athletes $(\mathrm{p}<$ $0.05)$. We also found differences in $\mathrm{BH}\left(\mathrm{F}_{5,125}=17.12, \mathrm{p}=\right.$
$0.00, \eta \mathrm{p}^{2}=0.41$; post-hoc test: $\mathrm{W}$ vs. J, K, F, T, and $\mathrm{Ki}(\mathrm{p}<$ $0.05)$; K vs. J, F $(\mathrm{p}<0.05)$ and $\mathrm{BM}\left(\mathrm{F}_{5,125}=9.59\right.$, $\mathrm{p}<0.00$, $\eta p^{2}=0.16$; post-hoc test: W vs. J, F, and Ki ( $\left.p<0.05\right)$; J vs. T ( $\mathrm{p}<0.05)$. Conversely, we did not find any significant difference in $\mathrm{BMI}\left(\mathrm{F}_{5,125}=1.84, \mathrm{p}=0.11, \eta \mathrm{p}^{2}=0.07\right)$ among the groups.

Body composition. The body composition profiles and differences in selected body composition parameters among the groups are presented in Table III. No significant differences among the groups were observed for relative values of $\mathrm{FM}_{\mathrm{p}}$, $\mathrm{FFM}_{\mathrm{rel}}$, and TBW $(\mathrm{p}>0.05)$. In contrast, we found significant differences for all other variables (Table III).

Table I. Performance levels of the included athletes.

\begin{tabular}{|c|c|}
\hline Sport & Performance level \\
\hline Judo & $\begin{array}{l}\text { Members of the national team, participants in international competitions and world cups, } \\
\text { several participants in the European Championship, one triple participant in the Olympic } \\
\text { Games }\end{array}$ \\
\hline Karate & $\begin{array}{l}\text { Members of the national team and the wider national team, participants in international } \\
\text { competitions and world cups }\end{array}$ \\
\hline Fencing & $\begin{array}{l}\text { Fencing -Épée. A group of } 14 \text { elite fencers - participants in the highest domestic } \\
\text { competitions, international competitions, and world cups; performance corresponding to } \\
\text { the first performance class determined a coording to the ranking of the Czech Fencing } \\
\text { Federation. Eight fencers were at the level of participation in domestic championships. }\end{array}$ \\
\hline Wrestling & $\begin{array}{l}\text { Members of the national team and the wider national team, most of them participants in } \\
\text { international competitions; both Greco-Roman and freestyle wrestlers are included }\end{array}$ \\
\hline Taekwondo & Members of the national team participating in international competitions \\
\hline Kickboxing & $\begin{array}{l}\text { Elite kickboxers were members of the national team, and in recent years they have } \\
\text { achieved significant domestic (medallists in national competitions) and international } \\
\text { success (world champion in WKA, WTKA, WKF, WKU association, European champion } \\
\text { in the WAKO association and medallists in international competitions). The participants' } \\
\text { specializations included light-contact, points fighting (semi-contact), and full-contact } \\
\text { disciplines. }\end{array}$ \\
\hline
\end{tabular}

WKA - World Kickboxing Association, WTKA - World Traditional Kickboxing Association, WKF - World Kickboxing Federation, WKU - World Kickboxing and Karate Union.

Table II. Basic anthropometric parameters of the athletes $(\mathrm{n}=132)$.

\begin{tabular}{|c|c|c|c|c|c|c|c|c|c|c|}
\hline \multirow{2}{*}{ Variables } & & \multirow{2}{*}{$\begin{array}{c}\text { Judo } \\
\mathrm{n}=19\end{array}$} & \multirow{2}{*}{$\begin{array}{l}\text { Karate } \\
\mathrm{n}=29\end{array}$} & \multirow{2}{*}{$\begin{array}{c}\text { Fencing } \\
\mathrm{n}=22\end{array}$} & \multirow{2}{*}{$\begin{array}{c}\text { Wrestling } \\
\mathrm{n}=25\end{array}$} & \multirow{2}{*}{$\begin{array}{c}\text { Taekwondo } \\
n=20\end{array}$} & \multirow{2}{*}{$\begin{array}{l}\text { Kickboxing } \\
\mathrm{n}=17\end{array}$} & \multicolumn{2}{|c|}{ ANOVA } & \multirow{2}{*}{ Post-hoc test } \\
\hline & & & & & & & & $F$ & $\eta p^{2}$ & \\
\hline Age (years) & $X(S D)$ & $\begin{array}{l}21.74 \\
(3.30)\end{array}$ & $\begin{array}{l}21.83 \\
(5.57)\end{array}$ & $\begin{array}{l}23.73 \\
(5.41)\end{array}$ & $\begin{array}{l}19.28 \\
(4.82)\end{array}$ & $\begin{array}{l}19.10 \\
(2.59)\end{array}$ & $\begin{array}{l}23.53 \\
(7.03)\end{array}$ & $4.06^{* *}$ & 0.14 & F vs. W, T \\
\hline Body height $(\mathrm{cm})$ & $\mathrm{X}(\mathrm{SD})$ & $\begin{array}{l}183.79 \\
(6.49)\end{array}$ & $\begin{array}{l}177.48 \\
(7.35)\end{array}$ & $\begin{array}{r}185.69 \\
(5.99)\end{array}$ & $\begin{array}{l}169.05 \\
(9.42)\end{array}$ & $\begin{array}{l}180.35 \\
(5.70)\end{array}$ & $\begin{array}{r}183.51 \\
(5.62)\end{array}$ & $17.12 * *$ & 0.41 & $\begin{array}{c}\text { W vs. J, K, F, T, Ki } \\
\text { Kvs. J, F }\end{array}$ \\
\hline Body mass (kg) & $X(S D)$ & $\begin{array}{c}83.38 \\
(11.65)\end{array}$ & $\begin{array}{l}74.57 \\
(9.97)\end{array}$ & $\begin{array}{l}78.68 \\
(9.46)\end{array}$ & $\begin{array}{l}68.27 \\
(14.16)\end{array}$ & $\begin{array}{c}72.16 \\
(12.52)\end{array}$ & $\begin{array}{c}80.80 \\
(11.82)\end{array}$ & $4.83 * *$ & 0.16 & W vs. J, F, Ki J vs. T \\
\hline BMI $\left(\mathrm{kg} \cdot \mathrm{m}^{-2}\right)$ & $X(S D)$ & $\begin{array}{l}24.63 \\
(2.43)\end{array}$ & $\begin{array}{l}23.61 \\
(2.38)\end{array}$ & $\begin{array}{l}22.86 \\
(2.97)\end{array}$ & $\begin{array}{l}23.70 \\
(3.29)\end{array}$ & $\begin{array}{l}22.10 \\
(3.10)\end{array}$ & $\begin{array}{l}23.92 \\
(2.86)\end{array}$ & 1.84 & 0.07 & \\
\hline
\end{tabular}


Segmental body fluid distribution. Analysis of variance revealed significant differences among the groups for all variables (Table IV). All of these variables were expressed as absolute values. Kickboxers showed the highest values of fluid in all variables.
Morphological differences. The findings for morphological differences were interesting (Table V). Morphological differences in the upper limbs were significantly greater in fencers $(0.20 \pm$ $0.11 \mathrm{l})$ than in judo $(0.20 \pm 0.11 \mathrm{l})$, karate $(0.10 \pm 0.07 \mathrm{l})$, wrestling $(0.06 \pm 0.06 \mathrm{l})$, and taekwondo athletes $(0.06 \pm 0.06 \mathrm{l})$.

Table III. Differences in body composition parameters among the groups.

\begin{tabular}{|c|c|c|c|c|c|c|c|c|c|c|}
\hline \multirow{2}{*}{\multicolumn{2}{|c|}{ Variables }} & Judo & Karate & Fencing & Wrestling & Taekwondo & Kickboxing & \multicolumn{3}{|l|}{ ANOVA } \\
\hline & & $\mathrm{n}=19$ & $\mathrm{n}=29$ & $\mathrm{n}=22$ & $\mathrm{n}=25$ & $\mathrm{n}=20$ & $\mathrm{n}=17$ & $F$ & $\eta p^{2}$ & \\
\hline $\mathrm{FM}(\%)$ & $\begin{array}{c}X \\
(\mathrm{SD})\end{array}$ & $\begin{array}{c}9.81 \\
(3.75)\end{array}$ & $\begin{array}{l}11.36 \\
(5.10)\end{array}$ & $\begin{array}{l}11.09 \\
(4.91)\end{array}$ & $\begin{array}{l}11.86 \\
(4.19)\end{array}$ & $\begin{array}{l}12.82 \\
(4.46)\end{array}$ & $\begin{array}{l}12.63 \\
(3.92)\end{array}$ & 1.06 & 0.04 & Post-hoc test \\
\hline FFM (kg) & $\begin{array}{l}\mathrm{X}) \\
(\mathrm{SD}\end{array}$ & $\begin{array}{l}74.58 \\
(9.91)\end{array}$ & $\begin{array}{l}65.91 \\
(8.08)\end{array}$ & $\begin{array}{l}69.71 \\
(6.24)\end{array}$ & $\begin{array}{c}59.92 \\
(12.07)\end{array}$ & $\begin{array}{c}62.91 \\
(10.96)\end{array}$ & $\begin{array}{l}70.21 \\
(7.21)\end{array}$ & $6.80 * *$ & 0.21 & $\begin{array}{c}\text { J vs. K, W, Ta } \\
\text { K vs. F. Ki }\end{array}$ \\
\hline $\mathrm{FFM}_{\text {rel }}$ & $\begin{array}{c}X \\
(\mathrm{SD})\end{array}$ & $\begin{array}{c}0,90 \\
(0.04)\end{array}$ & $\begin{array}{c}0.89 \\
(0.05)\end{array}$ & $\begin{array}{c}0.89 \\
(0.05)\end{array}$ & $\begin{array}{c}0.88 \\
(0.04)\end{array}$ & $\begin{array}{c}0.87 \\
(0.04)\end{array}$ & $\begin{array}{c}0.87 \\
(0.04)\end{array}$ & 0.86 & 0.03 & 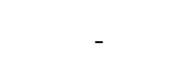 \\
\hline TBW (l) & $\begin{array}{c}X \\
(\mathrm{SD})\end{array}$ & $\begin{array}{l}54.97 \\
(7.42)\end{array}$ & $\begin{array}{l}47.59 \\
(5.85)\end{array}$ & $\begin{array}{l}49.61 \\
(4.49)\end{array}$ & $\begin{array}{l}44.06 \\
(8.87)\end{array}$ & $\begin{array}{l}45.82 \\
(8.17)\end{array}$ & $\begin{array}{l}51.45 \\
(5.95)\end{array}$ & $6.56 * *$ & 0.21 & $\begin{array}{c}\text { J vs. W, T W } \\
\text { vs. } \mathrm{Ki}\end{array}$ \\
\hline $\mathrm{TBW}_{\mathrm{p}}(\%)$ & $\begin{array}{c}\mathrm{X} \\
(\mathrm{SD})\end{array}$ & $\begin{array}{l}66.02 \\
(2.55)\end{array}$ & $\begin{array}{l}64.01 \\
(3.63)\end{array}$ & $\begin{array}{l}63.27 \\
(3.00)\end{array}$ & $\begin{array}{l}64.69 \\
(3.23)\end{array}$ & $\begin{array}{l}63.47 \\
(2.93)\end{array}$ & $\begin{array}{l}64.17 \\
(2.16)\end{array}$ & 2.13 & 0.08 & - \\
\hline ECW (l) & $\begin{array}{c}X \\
(\mathrm{SD})\end{array}$ & $\begin{array}{l}19.04 \\
(2.43)\end{array}$ & $\begin{array}{l}16.81 \\
(1.21)\end{array}$ & $\begin{array}{l}17.31 \\
(1.02)\end{array}$ & $\begin{array}{l}15.31 \\
(2.77)\end{array}$ & $\begin{array}{l}15.87 \\
(2.84)\end{array}$ & $\begin{array}{l}17.65 \\
(1.31)\end{array}$ & $8.57 * *$ & 0.26 & $\begin{array}{c}\text { J vs. K, W, Ta } \\
\text { W vs. F, Ki }\end{array}$ \\
\hline ICW(1) & $\begin{array}{c}X \\
(\mathrm{SD})\end{array}$ & $\begin{array}{l}35.93 \\
(5.55)\end{array}$ & $\begin{array}{l}30.78 \\
(4.76)\end{array}$ & $\begin{array}{l}32.30 \\
(3.55)\end{array}$ & $\begin{array}{l}28.75 \\
(6.75)\end{array}$ & $\begin{array}{l}29.96 \\
(6.11)\end{array}$ & $\begin{array}{l}33.73 \\
(4.79)\end{array}$ & $4.83 * *$ & 0.16 & J vs. K, W, T \\
\hline $\begin{array}{l}\text { Bone Mass } \\
(\mathrm{kg})\end{array}$ & $\begin{array}{c}X \\
(\mathrm{SD})\end{array}$ & $\begin{array}{c}4.34 \\
(0.68)\end{array}$ & $\begin{array}{c}3.27 \\
(0.38)\end{array}$ & $\begin{array}{c}3.45 \\
(0.27)\end{array}$ & $\begin{array}{c}3.40 \\
(0.86)\end{array}$ & $\begin{array}{c}3.14 \\
(0.51)\end{array}$ & $\begin{array}{l}3.59 \\
(0.45)\end{array}$ & $11.61 * *$ & 0.32 & $\begin{array}{c}\text { J vs. K, F, W, } \\
\text { T. Ki }\end{array}$ \\
\hline
\end{tabular}

FM - fat mass, FFM - fat free mass, FFMrel - relative value of fat free mass, TBW - total body water, TBWp - percentage of total body water, ECW extracellular water, ICW - intracellular water, $\mathrm{X}$ - arithmetic mean, SD - standard deviation, J - judo, K - karate, F - fencing, T - taekwondo, Ki kickboxing, $* *-p<0.01$.

Table IV.Segmental body fluid distribution and comparison among the groups.

\begin{tabular}{|c|c|c|c|c|c|c|c|c|c|c|}
\hline \multirow{2}{*}{\multicolumn{2}{|c|}{ Variables }} & \multirow{3}{*}{$\begin{array}{c}\text { Judo } \\
\mathrm{n}=19 \\
29.14 \\
(4.04)\end{array}$} & \multirow{3}{*}{$\begin{array}{l}\text { Karate } \\
\mathrm{n}=29 \\
33.42 \\
(3.78)\end{array}$} & \multirow{3}{*}{$\begin{array}{c}\begin{array}{c}\text { Fencing } \\
\mathrm{n}=22\end{array} \\
35.35 \\
(3.04)\end{array}$} & \multirow{3}{*}{$\begin{array}{c}\begin{array}{c}\text { W restling } \\
\mathrm{n}=25\end{array} \\
26.24 \\
(5.28)\end{array}$} & \multirow{3}{*}{$\begin{array}{c}\text { Taekwondo } \\
\mathrm{n}=20\end{array}$} & \multirow{3}{*}{$\begin{array}{c}\begin{array}{c}\text { Kickboxing } \\
\mathrm{n}=17\end{array} \\
36.44 \\
(3.57)\end{array}$} & \multicolumn{2}{|c|}{ ANOVA } & \multirow{2}{*}{ Post-hoc test } \\
\hline & & & & & & & & $F$ & $\eta p^{2}$ & \\
\hline Trunk (1) & $\begin{array}{c}X \\
(\mathrm{SD})\end{array}$ & & & & & & & $18.21 * *$ & 0.42 & W vs. K, F, T, Ki J vs. K, F, Ki \\
\hline NA (1) & $\begin{array}{c}X \\
(\mathrm{SD})\end{array}$ & $\begin{array}{c}3.99 \\
(0.63)\end{array}$ & $\begin{array}{c}3.81 \\
(0.71)\end{array}$ & $\begin{array}{c}4.00 \\
(0.58)\end{array}$ & $\begin{array}{c}3.13 \\
(0.77)\end{array}$ & $\begin{array}{c}3.38 \\
(1.01)\end{array}$ & $\begin{array}{c}4.40 \\
(0.62)\end{array}$ & $7.66^{* *}$ & 0.24 & W vs. J, K, F, Ki T vs. Ki \\
\hline PA (l) & $\begin{array}{c}X \\
(\mathrm{SD})\end{array}$ & $\begin{array}{c}4.01 \\
(0.66)\end{array}$ & $\begin{array}{c}3.86 \\
(0.73)\end{array}$ & $\begin{array}{c}4.16 \\
(0.59)\end{array}$ & $\begin{array}{r}3 . \\
(0 .\end{array}$ & $\begin{array}{r}3 . \\
(1 .\end{array}$ & $\begin{array}{c}4.44 \\
(0.70)\end{array}$ & $8.54 * *$ & 0.26 & W vs. J, K, F,KiT vs. F, Ki \\
\hline NL (l) & $\begin{array}{c}X \\
(\mathrm{SD})\end{array}$ & $\begin{array}{l}10.05 \\
(1.41)\end{array}$ & $\begin{array}{l}10.67 \\
(1.41)\end{array}$ & $\begin{array}{l}11.32 \\
(1.04)\end{array}$ & $\begin{array}{c}8.10 \\
(1.68)\end{array}$ & $\begin{array}{l}10.32 \\
(1.98)\end{array}$ & $\begin{array}{l}11.59 \\
(1.23)\end{array}$ & $15.53 * *$ & 0.38 & W vs. J, K, F, W, T, Ki \\
\hline PL (l) & $\begin{array}{c}X \\
\text { (SD) }\end{array}$ & $\begin{array}{l}10.15 \\
(1.51)\end{array}$ & $\begin{array}{l}10.88 \\
(1.29)\end{array}$ & $\begin{array}{l}11.42 \\
(1.00)\end{array}$ & $\begin{array}{c}8.22 \\
(1.73)\end{array}$ & $\begin{array}{l}10.56 \\
(1.87)\end{array}$ & $\begin{array}{l}11.71 \\
(1.07)\end{array}$ & $16.64 * *$ & 0.40 & W vs. J, K, F, W, T, Ki J vs. Ki \\
\hline
\end{tabular}

$\mathrm{NA}$ - non-preferred arm, PA - preferred arm, NL - non-preferred leg, PL - preferred leg, X - arithmetic mean, SD - standard deviation, J - judo, K karate, $\mathrm{F}$ - fencing, $\mathrm{T}-$ taekwondo, $\mathrm{Ki}-$ kickboxing, $* *-\mathrm{p}<0.01$.

Table V. Morphological differences among different athletes.

\begin{tabular}{ccccccccccc}
\hline \multicolumn{2}{c}{ Variables } & Judo & Karate & Fencing & Wrestling & Taekwondo & Kickboxing & \multicolumn{2}{c}{ ANOVA } & Post-hoc test \\
& $\mathrm{n}=19$ & $\mathrm{n}=29$ & $\mathrm{n}=22$ & $\mathrm{n}=25$ & $\mathrm{n}=20$ & $\mathrm{n}=17$ & $F$ & $\eta p^{2}$ & \\
\hline $\mathrm{UE}_{\text {diff }_{\mathrm{f}}}(\mathrm{l})$ & $\mathrm{X}$ & 0.08 & 0.10 & 0.20 & 0.06 & 0.06 & 0.12 & $9.07 * *$ & 0.27 & $\mathrm{~F}$ vs. J, K, W, T \\
& $(\mathrm{SD})$ & $(0.07)$ & $(0.07)$ & $(0.11)$ & $(0.06)$ & $(0.06)$ & $(0.11)$ & & & \\
$\mathrm{LE}_{\text {diff }}(\mathrm{l})$ & $\mathrm{X}$ & 0.14 & 0.28 & 0.21 & 0.16 & 0.29 & 0.26 & $4.06 * *$ & 0.14 & J vs. K, T K vs. W \\
& $(\mathrm{SD})$ & $(0.10)$ & $(0.13)$ & $(0.13)$ & $(0.14)$ & $(0.19)$ & $(0.16)$ & & &
\end{tabular}

UEdiff - paired differences between upper limbs, LEdiff - paired differences between lower limbs, X - arithmetic mean, SD - standard deviation, J judo, $\mathrm{K}$ - karate, $\mathrm{F}$ - fencing, $\mathrm{T}$ - taekwondo, $\mathrm{Ki}$ - kickboxing, ** $-\mathrm{p}<0.01$. 
In contrast, we detected the greatest morphological differences in athletes involved in sports that prioritize kicking (taekwondo, karate, kickboxing) or a standing fight position (fencers) in the lower limbs.
The comparison of paired differences between limbs (upper and lower limbs) revealed significantly greater fluid volume in the preferred arm compared to the non-preferred arm in karate and fencing athletes (Table VI). Moreover, we found significantly greater differences in favour of the preferred leg in all athletes.

Table VI. Paired differences between the lower and upper limbs in the observed groups.

\begin{tabular}{|c|c|c|c|c|c|c|c|c|c|}
\hline & \multirow{3}{*}{ Sport } & \multicolumn{5}{|c|}{ Paired Differences } & \multirow{3}{*}{$t$} & \multirow{3}{*}{ df } & \multirow{3}{*}{ Sig. (2-tailed) } \\
\hline & & \multirow{2}{*}{$X$} & \multirow{2}{*}{ SD } & \multirow{2}{*}{ Std. Error Mean } & \multicolumn{2}{|c|}{$95 \% \mathrm{CI}$ of the Difference } & & & \\
\hline & & & & & Lower & Upper & & & \\
\hline \multirow{6}{*}{$\begin{array}{l}\frac{b}{E} \\
\equiv \\
\bar{\Phi} \\
\vdots \\
\Xi\end{array}$} & Judo & -0.01 & 0.11 & 0.03 & -0.07 & 0.04 & -0.4 & 18 & 0.69 \\
\hline & Karate & -0.06 & 0.11 & 0.02 & -0.1 & -0.01 & -2.73 & 28 & 0.01 \\
\hline & Fencing & -0.15 & 0.17 & 0.04 & -0.23 & -0.08 & -4.24 & 21 & 0 \\
\hline & Wrestling & 0.01 & 0.08 & 0.02 & -0.02 & 0.05 & 0.72 & 24 & 0.48 \\
\hline & Taekwondo & 0.03 & 0.09 & 0.02 & -0.01 & 0.07 & 1.55 & 19 & 0.14 \\
\hline & Kickboxing & -0.04 & 0.16 & 0.04 & -0.12 & 0.05 & -0.9 & 16 & 0.38 \\
\hline \multirow{6}{*}{ 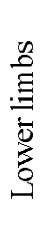 } & Judo & -0.1 & 0.19 & 0.04 & -0.19 & -0.01 & -2.35 & 18 & 0.03 \\
\hline & Karate & -0.22 & 0.19 & 0.04 & -0.29 & -0.14 & -6.07 & 28 & 0 \\
\hline & Fencing & -0.1 & 0.18 & 0.04 & -0.18 & -0.02 & -2.61 & 21 & 0.02 \\
\hline & Wrestling & -0.12 & 0.18 & 0.04 & -0.2 & -0.04 & -3.29 & 24 & 0 \\
\hline & Taekwondo & -0.24 & 0.24 & 0.05 & -0.36 & -0.13 & -4.58 & 19 & 0 \\
\hline & Kickboxing & -0.13 & 0.2 & 0.05 & -0.22 & -0.02 & -2.28 & 16 & 0.04 \\
\hline
\end{tabular}

$\mathrm{X}$ - arithmetic mean, $\mathrm{SD}$ - standard deviation, $\mathrm{t}$ - value of the testing criterion in a t-test; Sig. (2-tailed) - significance; CI- confidence interval, $\mathrm{df}$ - degrees of freedom.

\section{DISCUSSION}

Anthropometric parameters. The smallest and lightest athletes in our research group were wrestlers $(\mathrm{p}<0.05)$. The youngest athletes were fencers and taekwondo athletes. Values of basic anthropometric parameters were taken into account in the interpretation of the relative values of parameters identifying body composition quality.

Body composition. The body composition in all combat athletes was at an elite level. A low proportion of FM, with an exception in the case of competitors in heavier weight categories, and a high proportion of active mass (FFM and ICW) was found in all groups. Differences among the monitored groups were recorded as absolute values of FFM, TBW, ECW and ICW ( $\mathrm{p}<0.05)$. Relative values did not show any significant differences (Table III). Our findings were not in line with the available literature, which points to differences among different martial arts. Dopsaj et al. studied 112 male, high-level, senior-age athletes who were members of different national teams, including the Republic of Serbia team (62 judokas, 29 Greco-Roman wrestlers, and 21 karatekas). The authors identified the factors most associated with body structure indexes, through which specific changes in physical structure can be observed according to the branch of sport or type of combat sport. The authors presented the factors that are most associated with sport function. As the first isolated factor, percentage of skeletal mass $(0.34)$, body fat percentage $(0.28)$, and protein fat index $(0.26)(\mathrm{p}=0.000)$ explained 86.3 $\%$ of sport function. As the second factor, body mass (0.74), skeletal muscle index (0.73), protein mass index (0.72), BMI index (0.72), and FFM index (0.70) $(\mathrm{p}=0.030)$ explained 13.7 $\%$ of variability in measured body structure space.

The highest absolute value of FFM was found in judo athletes (Table III). Since success in judo is largely determined by strength, FFM together with compartment muscle mass predicts muscular efficiency and sports performance. The mean values of FFM suggest that judo and kickboxing athletes benefit from high FFM. The only other significant difference was detected for absolute FFM.

A lower proportion of FMp was found in judo athletes $(9.8 \pm 3.75 \%)$. Furthermore, in line with previous reports, all groups had a low proportion of FMp. Previous studies have presented the FMp in kickboxing elite athletes (6.1$11.4 \%$ ) (Ljubisavljevic et al.), amateur athletes (9-16\%) (Ouergui et al., 2014), boxers (9-16\%) (Chaabène et al., 2015), karate athletes (7.5-16.8 \%) (Chaabène et al., 2012), and judokas (7.4-14.7\%) (Franchini et al.; Mala et al., 2017). 
We did not find any significant differences in FM among the groups (Table III). The highest values of $\mathrm{FM}_{\mathrm{p}}^{\mathrm{p}}$ were observed in taekwondo athletes $(12.82 \pm 4.46 \%)$ and the lowest in judo athletes $(9.81 \pm 3.75 \%)$. Individual assessment of athletes revealed higher FMp in athletes with greater body mass and a tendency to be included in heavier weight categories (if stated by the rules). It has been reported that competitors in heavier weight categories have a higher percentage of FM. Low FM values and high FFM val-ues (Table III) confirmed the tendency toward elite performance in all athletes.

Fluid distribution. TBW accounted for $63-66 \%$ of body mass, while ICW accounted for $65 \%$ of TBW on average. The significant difference (Table IV) in TBW and its components (ICW and ECW) among the groups was the result of comparing the absolute values without taking $\mathrm{BH}$ and $\mathrm{BM}$ into account. As such, these differences do not indicate higher-quality BC in particular martial arts athletes. The lowest absolute values of fluid distribution in individual segments were found in wrestlers, which can be explained by the fact that these athletes had the lowest BM and BH. Due to the distribution of segmental body fluid, the specifics of particular martial arts were revealed. The highest proportion of body fluid in the lower limbs was found in kickboxers and fencers, whereas the lowest proportions of body fluid in the upper limbs were recorded in taekwondo athletes and wrestlers.

Morphological differences. Unilateral load is required in certain sports specializations (e.g., fencing, karate, and kickboxing). If one side of the body dominates over the other because of the requirements of specific movements (e.g., kicking in kickboxing), different forms of asymmetry can appear (morphological, neuromuscular). These sports disciplines can be related to asymmetry due to tissue adaptation resulting in bilateral asymmetry (e.g., fluid distribution or strength differences between the limbs). Asymmetry is a consequence of extended lateral training which is used in many martial arts, particularly by those individuals who start to compete internationally. These combat athletes begin to develop their Tokui-Waza (special technique).

The most "symmetrical" sport included in the current study is judo, as the morphological differences in the upper limbs $(0.08 \pm 0.071)$ and morphological differences in the lower limbs $(0.14 \pm 0.101)$ were the lowest among the groups. In contrast, fencing appears to be the least symmetrical sport, since morphological differences in the upper limbs were significantly greater than those in judo, karate, wrestling, and taekwondo athletes (Table V).

This result is in accordance with the research of
Krstulovic et al., who tested 28 female judokas (aged $21.0 \pm 2.3$ years, Croatian national team) and reported differences in morphological, functional, and dynamic asymmetry between elite and sub-elite competitors. Statistically significant differences in the coefficients of asymmetry between elite and sub-elite female judokas were recorded in only two variables of dynamic asymmetry (maximum dynamometric force and seat shot put test). The authors reported no significant difference in coefficients assessing morphological asymmetry between elite and subelite female judokas. Furthermore, they presented relatively low levels of asymmetry in morphological variables in both groups of female judokas (0.4-1.6\% in elite judokas vs. $0.7-1.6 \%$ in sub-elite judokas).

In judokas, we observed a tendency to preferentially use one side of the body in a voluntary act. This tendency characterizes the lateral preference. This lateralization has been suggested to be only 10-20\% dependent on genetics. Similarly, in judo, when using the right grip with the dominant hand, right stance-dominant judokas usually operate with the left leg as the supporting leg and the right leg used as the "execution" or attacking leg. The right or attacking leg is also positioned closer to the opponent and is therefore more frequently attacked with hooking and sweeping techniques with the intention of throwing the opponent off balance. This mostly occurs in the scenario of two right grip fighters facing each other. Therefore, the execution leg performs more work than the supporting leg, which could lead to imbalance between the muscles of the left and right sides.

When comparing paired differences between the lower and upper limbs, we recorded a significant difference in asymmetry in judo athletes $(\mathrm{p}<0.05)$. The reason for this is likely long-lasting and regular training sessions combined with the intensive asymmetrical muscle loads associated with the sport, which may cause different types of overloads and lead to different motor organ injuries and deformations (Barczyk et al., 2012). The greatest morphological differences in the lower limbs were found in athletes engaged in kicking forms (taekwondo, karate, kickboxing) or in those who had a preferred standing fight position (fencers).

When assessing paired differences between the lower and upper limbs among the groups, fencing and karate appeared to be the most asymmetrical sports. Asymmetry in both the lower and upper limbs was found (Table VI). Even everyday activities, through the tendency for a greater functional use of the dominant limb in relation to the nondominant limb, may contribute to morphological asymmetry (Roy et al., 1994). In asymmetrical sporting disciplines (e.g., fencing), a certain level of morphological asymmetry often 
develops due to the physical and neurological demands of the locomotor apparatus of the dominant limb in relation to the non-dominant limb, which can even lead to ossification of some parts of the body in elite athletes (Krzykala).

To correctly perform a particular technique (e.g., high kicks in karate, kickboxing), an optimal range of joint mobility is required. Muscle imbalance and shortening may limit the range of joint mobility and can become a limiting factor for performing some movements (kicks and strikes) in karate. The reasons for muscle imbalance are unilateral overloading, overloading in a karateka's stance, and the absence of regular compensation exercises in training sessions. In the lower limbs, we found the greatest morphological difference in athletes preferring kicking (taekwondo, karate, and kickboxing) or standing fight positions (fencers). In all groups, we observed significant differences between the preferred and non-preferred lower limb. In terms of paired differences between the lower limbs, judo was considered an asymmetric sport (Table VI) $(\mathrm{p}=0.04)$. Judo can be categorized as an asymmetric sport, in which the combat techniques are performed in a certain direction that dominantly engages the muscles of either the left or right side of the body (Krstulovic et al.). Asymmetry in judo can be explained by the fact that right hand-dominant judokas usually fight with a right-dominant stance. The right hand is usually used to grab the lapel or sleeve of a judogi to start the attack. It can also be the leading upper arm in the right-versus-right stance scenario. The right hand is also the most activated during the kuzushi phase, when one fighter attempts to throw their opponent off balance. Mala et al. (2017) reported that in adolescent judo athletes, when personal techniques (Tokui-Waza) are being selected, the techniques are unilaterally executed and that when one limb becomes more preferred during Ashi-Waza (leg techniques), morphological asymmetry can develop in the proportion of muscle mass in upper and lower limbs. The authors monitored 39 adolescent judo athletes (age $12.08 \pm 1.47$ years) and reported that the bilateral comparison of the muscle mass proportion between the preferred and nonpreferred limbs showed a significantly higher proportion of muscle mass in the preferred upper and lower limbs in boys $(\mathrm{p}<0.01)$.

\section{CONCLUSIONS}

This study compared BC among competitors in six different martial arts. The study revealed morphological differences among the groups as well as between paired limbs. The specific principles of asymmetric and uncompensated activities in these sports disciplines may lead to asymmetry (morphological, functional, etc.) and are linked to tissue adaptation resulting in bilateral asymmetry. The prevalence and reasons for asymmetry may be induced and subsequently accelerated by unilaterally focused and insufficiently compensated sports activity (kicking, pushing, hitting, and jumping). Limb preferences (handedness and footedness) occur in sports as a part of adaptive processes and are even desirable when executing manipulations. However, from the perspective of morphology, physiology, and the level of physical ability, these differences should be minimized using proper compensatory exercises.

These data may be useful to other researchers performing comparative studies and may serve as a basis for the assessment of elite martial arts athletes. The results may be beneficial to athletes, coaches, athletic trainers, physiotherapists, doctors, and other clinical staff involved in elite martial arts. The forms of morphological asymmetry detected in this study should be eliminated using proper compensatory exercises.

\section{ACKNOWLEDGEMENTS}

This research was supported by GACR 16-21791S, UNCE HUM/032, SVV 2017-2019-260466

MALA, L.; MALY, T.; CABELL, L.; CECH, P; HANK, M.; COUFALOVA, K. \& ZAHALKA, F. Composición corporal y asimetría morfológica de miembros en competidores en seis artes marciales. Int. J. Morphol., 37(2):568-575, 2019.

RESUMEN: El objetivo de este estudio fue comparar la composición corporal (CC) y las diferencias de simetría morfológica entre atletas de élite $(\mathrm{n}=132)$ en seis artes marciales (judo, karate, esgrima, lucha, taekwondo, kickboxing). Se utilizó el análisis multivariado de varianzas (MANOVA) para comparar las siguientes variables: valor absoluto (FFM) y relativo $\left(\mathrm{FFM}_{\mathrm{rel}}\right)$ de la masa libre de grasa, porcentaje de masa grasa $\left(\mathrm{FM}_{\mathrm{p}}\right)$, masa ósea, masa proteica, tasa metabólica basal, absoluto (TBW) y el valor porcentual $\left(\mathrm{TBW}_{\mathrm{p}}\right)$ de líquido corporal total, la proporción segmentaria de la masa muscular, el ángulo de fase y las diferencias porcentuales entre los miembros superiores e inferiores. MANOVA reveló diferencias significativas en BC entre los gru$\operatorname{pos}\left(\lambda=0,01, \mathrm{~F}_{104,256}=10,01, \mathrm{p}<0,01, \eta \mathrm{p}^{2}=0,67\right)$. No se observaron diferencias significativas para $\mathrm{FM}_{\mathrm{p}}, \mathrm{FFM}_{\mathrm{rel}}$ y TBW $(\mathrm{p}>0,05)$. En todas las demás variables de $\mathrm{BC}$, se detectaron diferencias significativas $(\mathrm{p}<0,05)$. La comparación de las diferencias pareadas entre los miembros reveló un volumen de líquido significativamente mayor en el brazo dominante en comparación con el brazo no dominante en karate y atletas de esgrima. Se detectaron diferencias significativas $(p<0,05)$ a favor de la pierna dominante en todos los atletas de combate. Este estudio reveló diferencias morfológicas 
entre los practicantes de diferentes artes marciales y entre las miembros emparejadas.

PALABRAS CLAVE: Deportes de combate; Asimetrías morfológicas; Deportistas de élite; Masa grasa; Masa libre de grasa.

\section{REFERENCES}

Barczyk, K.; Bankosz, Z. \& Derlich, M. Body postures and asymmetries in frontal and transverse planes in the trunk area in table tennis players. Biol. Sport, 29(2):127-32, 2012.

Calbet, J. A.; Moysi, J. S.; Dorado, C. \& Rodríguez, L. P. Bone mineral content and density in professional tennis players. Calcif. Tissue Int., 62(6):491-6, 1998.

Chaabène, H.; Hachana, Y.; Franchini, E.; Mkaouer, B. \& Chamari, K. Physical and physiological profile of elite karate athletes. Sports Med., 42(10):829-43, 2012.

Chaabène, H.; Tabben, M.; Mkaouer, B.; Franchini, E.; Negra, Y.; Hammami, M.; Amara S.; Chaabène, R. B. \& Hachana, Y. Amateur boxing: physical and physiological attributes. Sports Med., 45(3):337$52,2015$.

Dopsaj, M.; Markovic, M.; Kasum, G.; Jovanovic, S.; Koropanovski, N.; Vukovic, M. \& Mudric, M. Discrimination of different body structure indexes of elite athletes in combat sports measured by multi frequency bioimpedance method. Int. J. Morphol., 35(1):199-207, 2017.

Franchini E.; Del Vecchio, F. B.; Matsushigue, K. A. \& Artioli, G. G. Physiological profiles of elite judo athletes. Sports Med., 41(2):14766, 2011.

Kannus, P.; Haapasalo, H.; Sankelo, M.; Sievänen, H.; Pasanen, M.; Heinonen, A.; Oja, P. \& Vuori, I. Effect of starting age of physical activity on bone mass in the dominant arm of tennis and squash players. Ann. Intern. Med., 123(1):27-31, 1995.

Kasum, G. \& Dopsaj, M. Descriptive profile of body structure of top grecoroman style wrestlers defined with method of multichannel bioelectric impendance. Sport Logia, 8(2):123-31, 2012.

Krstulovic, S.; Kuvacic, G. \& Erceg, M. Morphological, functional and dynamical asymmetry in female judokas. Acta Kinesiol., 11(2):12-8, 2017.

Krzykala, M. Dxa as a Tool for the Assessment of Morphological Asymmetry in Athletes. In: Maghraoui, A. (Ed.). Dual Energy X-Ray Absorptiometry. Rijeka, InTech, 2012.

Ljubisavljevic, M.; Amanovic, D.; Buncic, V. \& Simic, D. Differences in morphological characteristics and functional abilities with elite and subelite kick boxers. Sport Sci., 8 Suppl. 2:59-64, 2015.

Mala, L.; Maly, T.; Camirelli, R.; Dornowski, M.; Zahalka, F.; Petr, M.; Hrasky, P. \& Bujnovsky, D. Gender differences in strength lateral asymmetries, limbs morphology and body composition in adolescent judo athletes. Arch. Budo, 13:377-85, 2017.

Mala, L.; Maly, T.; Zahalka, F. \& Bunc, V. Fitness Assessment: Body Composition. Prague, Karolinum Press, 2014.

Mala, L.; Maly, T.; Zahalka, F. \& Dragijsky, M. Changes in body composition due to weight reduction by elite youth judo athletes in short period pre-competition. Arch. Budo. Sci. Martial Art Extrem. Sports, 12(1):197-203, 2016

Mala, L.; Maly, T.; Zahalka, F.; Bunc, V.; Kaplan, A.; Jebavy, R. \& Tuma, M. Body composition of elite female players in five different sports games. J. Hum. Kinet., 45:207-15, 2015.

Mala, L.; Maly, T.; Zahalka, F.; Heller, J.; Hrasky, P. \& Vodicka, P. Differences in the morphological and physiological characteristics of senior and junior elite Czech judo athletes. Arch. Budo, 11:217-26, 2015.

Maly, T.; Mala, L.; Zahalka, F.; Hank, M. \& Simkova, M. Muscular strength of knee extensors and flexors and bilateral and ipsilateral ratio in elite male kickboxers. Arch. Budo, 13:107-16, 2017.

Maly, T.; Zahalka, F. \& Mala, L. Unilateral and ipsilateral strength asymmetries in elite youth soccer players with respect to muscle group and limb dominance. Int. J. Morphol., 34(4):1339-44, 2016.

Ouergui, I.; Hssin, N.; Haddad, M.; Padulo, J.; Franchini, E.; Gmada, N. \& Bouhlel, E. The effects of five weeks of kickboxing training on physical fitness. Muscles Ligaments Tendons J., 4(2):106-13, 2014.

Rauter, S.; Vodicar, J. \& Simenko, J. Body asymmetries in young male road cyclists. Int. J. Morphol., 35(3):907-12, 2017.

Reilly, T. Fitness Assessment. In: Reilly, T. \& Williams, A. M. (Eds.). Science and Soccer. London, E. \& F. Spon, 1996. pp.25-50.

Roy, T. A.; Ruff, C. B. \& Plato, C. C. Hand dominance and bilateral asymmetry in the structure of the second metacarpal. Am. J. Phys. Anthropol., 94(2):203-11, 1994.

Sutton, L.; Scott, M.; Wallace, J. \& Reilly, T. Body composition of English Premier League soccer players: influence of playing position, international status, and ethnicity. J. Sports Sci., 27(10):1019-26, 2009.

Vad, V. B.; Bhat, A. L.; Basrai, D.; Gebeh, A.; Aspergren, D. D. \& Andrews J. R. Low back pain in professional golfers: the role of associated hip and low back range-of-motion deficits. Am. J. Sports Med., 32(2):494$7,2004$.

\author{
Corresponding author: \\ Lucia Mala, PhD \\ Sport Research Centre \\ Faculty of Physical Education and Sport \\ Charles University \\ Jose Martiho 31 \\ 16252 Prague 6 \\ CZECH REPUBLIC
}

Email: lucilali@yahoo.de

Received: 07-12-2018

Accepted: 28-01-2019 